\title{
Résumé : La maladie de Lyme au Canada - Cadre fédéral
}

\section{Centre des maladies infectieuses d'origine alimentaire, environnementale et zoonotique ${ }^{1 *}$}

\section{Résumé}

La Loi sur le cadre relatif à la maladie de Lyme exigeait que le gouvernement fédéral définisse un cadre fédéral relatif à la maladie de Lyme. Pour ce faire, l'Agence de la santé publique du Canada a organisé une conférence, à laquelle a participé un vaste éventail d'intervenants qui ont fait part de leurs expériences relatives à la maladie de Lyme et discuté des connaissances et des recherches actuelles. Une version préliminaire du cadre a été publiée sur le site Canada.ca pour une période de consultation publique de 30 jours. Le rapport final, intitulé La maladie de Lyme au Canada - Cadre fédéral, a été publié en mai 2017. Le cadre comporte les trois piliers suivants : surveillance; éducation et sensibilisation; lignes directrices et pratiques exemplaires. La mise en œuvre exigera la participation et la collaboration de tous les intervenants et de tous les ordres de gouvernement, le gouvernement du Canada s'engageant à collaborer avec des partenaires nationaux et internationaux pour l'échange des pratiques exemplaires en matière de prévention, de diagnostic et de traitement de la maladie de Lyme.

\section{Affiliation}

${ }^{1}$ Agence de la santé publique du Canada, Ottawa (Ontario)

${ }^{*}$ Correspondance : maladie lyme_disease@phac-aspc.gc.ca

Citation proposée : Centre des maladies infectieuses d'origine alimentaire, environnementale et zoonotique, Agence de la santé publique du Canada. Résumé : La maladie de Lyme au Canada - Cadre fédéral. Relevé des maladies transmissibles au Canada. 2017;43(10):239-42. https://doi.org/10.14745/ccdr.v43i10a04f

\section{Introduction}

La Loi sur le cadre fédéral relatif à la maladie de Lyme, qui a reçu la sanction royale le 16 décembre 2014, exigeait que le gouvernement fédéral élabore un cadre fédéral concernant la maladie de Lyme qui assurerait la cohésion des efforts relatifs à la surveillance, à l'éducation et à la sensibilisation, ainsi qu'aux lignes directrices et des meilleures pratiques. Afin d'orienter I'élaboration de ce cadre, I'Agence de la santé publique du Canada, au nom de la ministre de la Santé, a tenu une conférence à laquelle ont participé plus de 500 personnes. Des patients atteints de la maladie de Lyme, leurs familles et d'autres intervenants ont fait part de leurs expériences relatives à la maladie de Lyme, alors que des experts en la matière ont fourni des renseignements sur les connaissances et les recherches actuelles en ce qui concerne le traitement, la prévention, le diagnostic et la prise en charge de la maladie de Lyme. En outre, à des fins de rétroactions et d'amélioration, une version préliminaire du cadre a été publiée sur le site Canada.ca pour une période de consultation publique de 30 jours. Le rapport intégral, intitulé La maladie de Lyme au Canada - Cadre fédéral, a été publié le 30 mai 2017 et est accessible en ligne (1). Ce cadre constitue une première étape dans l'orientation des interventions concrètes dans les régions où le gouvernement fédéral a un rôle à jouer. Le présent document est un résumé du rapport intégral.

\section{Contexte}

Le Canada compte une quarantaine d'espèces de tiques. Parmi celles-ci, seules quelques-unes sont capables de transmettre des pathogènes, notamment des bactéries et des virus, ayant le potentiel de cause des maladies chez l'humain.

La maladie de Lyme est l'affection transmissible par les tiques la plus fréquente au Canada; elle est causée par la bactérie Borrelia burgdorferi. Cette bactérie se transmet aux humains par la morsure de la tique à pattes noires et de la tique occidentale à pattes noires.

La maladie de Lyme est présente principalement dans les régions, ou près de celles-ci, où des populations de tiques infectées sont établies. Les tiques adultes sont environ de la taille d'une graine de sésame, et les tiques au stade nymphal (immatures) sont environ de la taille d'une graine de pavot. C'est pour cette raison qu'une personne peut ne pas savoir qu'une tique est accrochée à sa peau. Les personnes qui effectuent des activités professionnelles ou récréatives, comme le camping ou la randonnée, dans les zones forestières ou semi-forestières (ou à proximité de ces dernières) où l'on trouve des tiques sont les plus exposées à la transmission par morsure. L'exposition aux tiques peut avoir lieu dans d'autres circonstances, notamment en jardinant, en jouant au golf ou en promenant son chien, si ses activités sont effectuées dans des régions où l'on trouve des tiques. Les tiques porteuses de la maladie de Lyme sont actives une grande partie de l'année; toutefois, les morsures entraînant une infection chez les humains sont bien plus courantes pendant les mois de printemps et d'été.

En 2016, on a nouvellement confirmé le diagnostic de la maladie de Lyme chez 987 Canadiens (2). Le nombre de cas est en croissance constante depuis que la maladie de Lyme est devenue à déclaration obligatoire à l'échelle nationale, en 2009. 
Toutefois, bien que des progrès en matière de connaissances et de diagnostic aient été accomplis au fil du temps, il est probable que la maladie soit sous-déclarée. Le gouvernement fédéral ainsi que les provinces et territoires continuent de collaborer afin de brosser le portrait le plus exact de la maladie de Lyme au Canada.

\section{Responsabilité}

Le gouvernement du Canada joue un rôle de chef de file national dans la prévention et le contrôle de la propagation de la maladie, et contribue ainsi à réduire le risque que présentent les maladies infectieuses pour les Canadiens. II remplit ce rôle en effectuant un suivi et une surveillance des menaces de maladies infectieuses, en entreprenant des recherches, en favorisant les comportements sains, et en facilitant le transfert des connaissances et l'innovation.

Plusieurs ministères et organismes du gouvernement fédéral luttent contre la maladie de Lyme au Canada :

- Agence de la santé publique du Canada

- Instituts de recherche en santé du Canada

- Santé Canada

- Ministère de la Défense nationale et Forces armées canadiennes

- Parcs Canada

Au Canada, la prévention et le contrôle de la maladie de Lyme nécessitent une collaboration entre tous les ordres du gouvernement, ainsi qu'avec les organisations non gouvernementales. Comme l'indiquent les dispositions de la Loi canadienne sur la santé, il incombe principalement aux provinces et aux territoires d'offrir des services de soins de santé et des activités de santé publique. Les autorités de santé publique provinciales et territoriales et les autorités autochtones de santé publique entreprennent des activités de prévention et de lutte propres à leurs territoires de compétence. Dans certaines provinces et certains territoires, ce travail est effectué en collaboration avec les universités et d'autres professionnels de la santé et organisations non gouvernementales.

\section{Mesures de santé publique}

Tous les intervenants, y compris les patients et leurs représentants, les fournisseurs de soins de santé et les autorités de santé publique, reconnaissent l'importance des approches fondées sur des données probantes pour la santé publique et la pratique de la médecine. De même, de nombreuses personnes conviennent que d'autres recherches sont nécessaires pour combler les lacunes en matière de données probantes qui existent pour la prévention, le contrôle, le diagnostic et le traitement de la maladie de Lyme.

Dans le cadre de la première étape en vue de corriger les lacunes en matière de données probantes, le gouvernement du Canada investira de nouveaux fonds afin de combler les lacunes en matière de recherche sur la maladie de Lyme, et continuera à collaborer avec les partenaires nationaux et internationaux pour l'échange des pratiques exemplaires en matière de prévention, de diagnostic et de traitement de la maladie de Lyme.
D'autres nouvelles interventions liées au cadre correspondent au rôle du gouvernement fédéral en ce qui a trait à la surveillance, à l'éducation et la sensibilisation et aux lignes directrices et pratiques exemplaires. La mise en œuvre nécessitera la participation et la collaboration des patients touchés par la maladie de Lyme, des groupes de patients, des fournisseurs de soins de santé, des responsables de la santé publique, des chercheurs experts, ainsi que des gouvernements fédéral, provinciaux et territoriaux.

\section{Surveillance}

La surveillance est essentielle pour comprendre le risque pour les Canadiens, et est le fondement de l'approche en matière de santé publique. La surveillance de la maladie de Lyme comprend les mesures suivantes : surveiller à la fois la répartition et la propagation des tiques porteuses de la bactérie $B$. burgdorferi, et effectuer un suivi des cas humains à l'échelle nationale.

Les autorités de santé publique fédérales et provinciales continueront de s'appuyer sur les activités de surveillance au moyen de l'intégration et de la diffusion des méthodes novatrices et des pratiques exemplaires pour la surveillance chez les humains.

En outre, la collecte de données de surveillance sur les personnes au Canada qui ne répondent pas à la définition de cas probable ou confirmé de la maladie de Lyme, mais qui présentent divers symptômes correspondant à la maladie de Lyme ou à des maladies semblables, sera lancée.

L'augmentation de la répartition et du nombre de personnes touchées par cette maladie au Canada a des répercussions financières sur le système de soins de santé. Une analyse des coûts associés à la maladie de Lyme sera entreprise, y compris les coûts directs et indirects, dans la mesure du possible. De plus, un système national de surveillance de la transmission par les tiques sera mis au point; il comprendra la maladie de Lyme et d'autres infections transmissibles par les tiques possibles.

\section{Éducation et sensibilisation}

Il convient d'accroître les efforts visant à améliorer les activités éducatives liées à la maladie de Lyme afin qu'elles soient plus efficaces et plus facilement accessibles aux Canadiens et aux professionnels de la santé de première ligne, à l'appui des gouvernements provinciaux et territoriaux et des autres efforts.

Il sera essentiel de renforcer la participation des intervenants et les partenariats afin que les campagnes éducatives et de sensibilisation soient couronnées de succès. L'élaboration de matériel éducatif sur la détection et le diagnostic précoces, qui met l'accent sur les groupes à risque élevé, servira à aider les professionnels de la santé de première ligne et les autorités de santé publique dans le cadre de la prévention et du diagnostic précoce de la maladie de Lyme, au moyen d'une approche fondée sur des données probantes et axée sur le patient. Étant donné que la prévention est un élément essentiel de l'approche en matière de santé publique pour les maladies infectieuses, une campagne nationale d'éducation et de sensibilisation sur les tiques et la maladie de Lyme sera mise en place, en collaboration avec des partenaires, pour compléter les efforts de sensibilisation visant à réduire le risque de contracter la 
maladie de Lyme et pour guider l'intervention, le diagnostic et le traitement précoces.

\section{Lignes directrices et pratiques exemplaires}

Il sera essentiel de disposer de lignes directrices et de pratiques exemplaires qui sont fondées sur des données probantes et qui ciblent efficacement des groupes déterminés. On devra aborder, entre autres, la prévention, le diagnostic, le traitement et la recherche.

À I'heure actuelle, la meilleure façon de se protéger contre la maladie de Lyme est de prévenir les morsures de tiques ou, en cas de morsure, de réduire au minimum le risque d'infection par le retrait de la tique en temps opportun et de façon efficace (3). Des programmes de prévention et de sensibilisation sont mis en œuvre par les responsables locaux de la santé publique et d'autres fournisseurs de soins de santé et de soins vétérinaires, afin d'accroître la sensibilisation aux risques que présente la maladie de Lyme et aux mesures nécessaires pour se protéger des morsures de tiques.

Le diagnostic de l'infection par la maladie de Lyme à un stade précoce, établi par un médecin ou une infirmière praticienne, est principalement clinique et s'appuie sur des symptômes ainsi que sur des antécédents de possible exposition à des tiques, y compris les antécédents de voyage. Le diagnostic est difficile par le fait que ce ne sont pas tous les patients qui présenteront des symptômes à un stade précoce. Les analyses en laboratoire actuelles, qui examinent les anticorps, sont plus efficaces pour la maladie de Lyme non traitée, à un stade avancé, et après que le patient a développé une réponse immunitaire. En raison de préoccupations au sujet des faux négatifs, certains patients se rendent dans des laboratoires privés pour effectuer les analyses. Grâce à l'élaboration et à la disponibilité de meilleures options d'analyses en laboratoire, certains patients pourraient délaisser leur pratique actuelle, qui consiste à effectuer les analyses auprès de laboratoires privés et à but lucratif qui pourraient ne pas utiliser des méthodes d'analyse normalisées. Il est reconnu que des tests précis et plus sensibles pour la maladie de Lyme sont nécessaires.

Il reste encore des lacunes en matière de données probantes qui peuvent être éclairées par davantage de recherches sur les options de traitement. Par exemple, certaines personnes présentent des symptômes qui persistent plus de six mois après un traitement, ce que certains médecins appellent le syndrome de Lyme post-traitement ou le syndrome post-maladie de Lyme. D'autres patients présentent différents symptômes chroniques correspondant à la maladie de Lyme ou à des maladies semblables, que l'on appelle parfois maladie de Lyme chronique; celle-ci n'est pas reconnue par la majorité des professionnels de la santé au Canada. Pour accroître la confusion, les lignes directrices actuelles relatives au traitement de la maladie de Lyme sont élaborées par des organismes professionnels médicaux et scientifiques, et s'appuient sur les meilleures données probantes disponibles dans le monde. Au pays, l'Association pour la microbiologie médicale et I'infectiologie Canada a approuvé et encouragé I'utilisation des lignes directrices sur le traitement de la maladie de Lyme élaborées par I'Infectious Diseases Society of America (IDSA), qui représente des médecins, des scientifiques et d'autres professionnels de la santé spécialisés en maladies infectieuses
(4). Par conséquent, les lignes directrices de I'IDSA sont utilisées par la majorité de la communauté médicale. Cependant, il existe un petit nombre de professionnels de la santé de première ligne qui suivent les lignes directrices élaborées par l'International Lyme and Associated Diseases Society (ILADS), une société de santé médicale multidisciplinaire consacrée au diagnostic et au traitement de la maladie de Lyme et des maladies connexes. Les recommandations en matière de traitement formulées dans les lignes directrices de l'ILADS sont différentes de celles de I'IDSA, particulièrement en ce qui a trait à l'utilisation des antibiotiques. Il est nécessaire d'approfondir la recherche sur les meilleures méthodes de traitement.

À l'avenir, le gouvernement du Canada collaborera avec les partenaires internationaux en matière de santé publique pour échanger des pratiques exemplaires, et ces dernières seront communiquées à tous les intervenants. Les professionnels de la santé de première ligne et les laboratoires provinciaux continueront d'être soutenus dans le diagnostic en laboratoire de la maladie de Lyme. Nos partenaires, y compris les organismes de réglementation de soins de santé provinciaux et territoriaux, seront consultés à propos des approches novatrices et fondées sur des données probantes pour répondre aux besoins des patients, et un réseau national de recherche sur la maladie de Lyme sera établi pour s'appuyer sur des recherches existantes en vue de combler les lacunes en matière de données probantes et de mobiliser des experts cliniciens, des chercheurs et des groupes de patients.

\section{Conclusion}

Afin de s'assurer que les efforts continus pour lutter contre la maladie de Lyme soient fondés sur des données probantes et que le gouvernement du Canada poursuive ses progrès en matière de prévention et de contrôle de la propagation de la maladie de Lyme, l'Agence de la santé publique du Canada examinera le présent cadre dans les cinq années suivant sa publication.

\section{Remerciements}

L'Agence de la santé publique du Canada aimerait remercier tous ceux qui ont contribué, grâce à leur temps et leur expertise, à l'élaboration du Cadre fédéral relatif à la maladie de Lyme.

\section{Financement}

L'élaboration du Cadre fédéral relatif à la maladie de Lyme a été financée par le gouvernement du Canada.

\section{Références}

1. Gouvernement du Canada. La maladie de lyme au Canada - Cadre fédéral. Ottawa : Gouvernement du Canada. Mai 2017. https://www.canada.ca/fr/sante-publique/services/ 
publications/maladies-et-affections/maladie-lyme-canadacadre-federal.html

2. Surveillance de la maladie de Lyme: Nombre de cas signalés de la maladie de Lyme. Ottawa: Gouvernement du Canada; [Consulté le 4 juill 2017]. https://www.canada.ca/fr/santepublique/services/maladies/maladie-lyme/surveillancemaladie-lyme.html\#a2

3. Prévention de la maladie de Lyme. Ottawa: Gouvernement du Canada. https://www.canada.ca/fr/sante-publique/ services/maladies/maladie-lyme/prevention-maladie-lyme. html
4. Wormser GP, Dattwyler RJ, Shapiro ED, Halperin JJ, Steere AC, Klempner MS, Krause PJ, Bakken JS, Strle F, Stanek G, Bockenstedt L, Fish D, Dumler JS, Nadelman RB. The clinical assessment, treatment, and prevention of Lyme disease, human granulocytic anaplasmosis, and babesiosis: clinical practice guidelines by the Infectious Diseases Society of America. Clin Infect Dis. 2006 Nov 1;43(9):1089-134. Epub 2006 Oct 2. Erratum in: Clin Infect Dis. 2007 Oct 1;45(7):941. DOI (http://dx.doi.org/10.1086/508667). PubMed (https:// www.ncbi.nlm.nih.gov/entrez/query.fcgi?cmd=Retrieve\&db= PubMed\&list_uids=17029130\&dopt=Abstract).

\section{PASSEZ LE MOT À PROPOS DE LA GRIPPE}

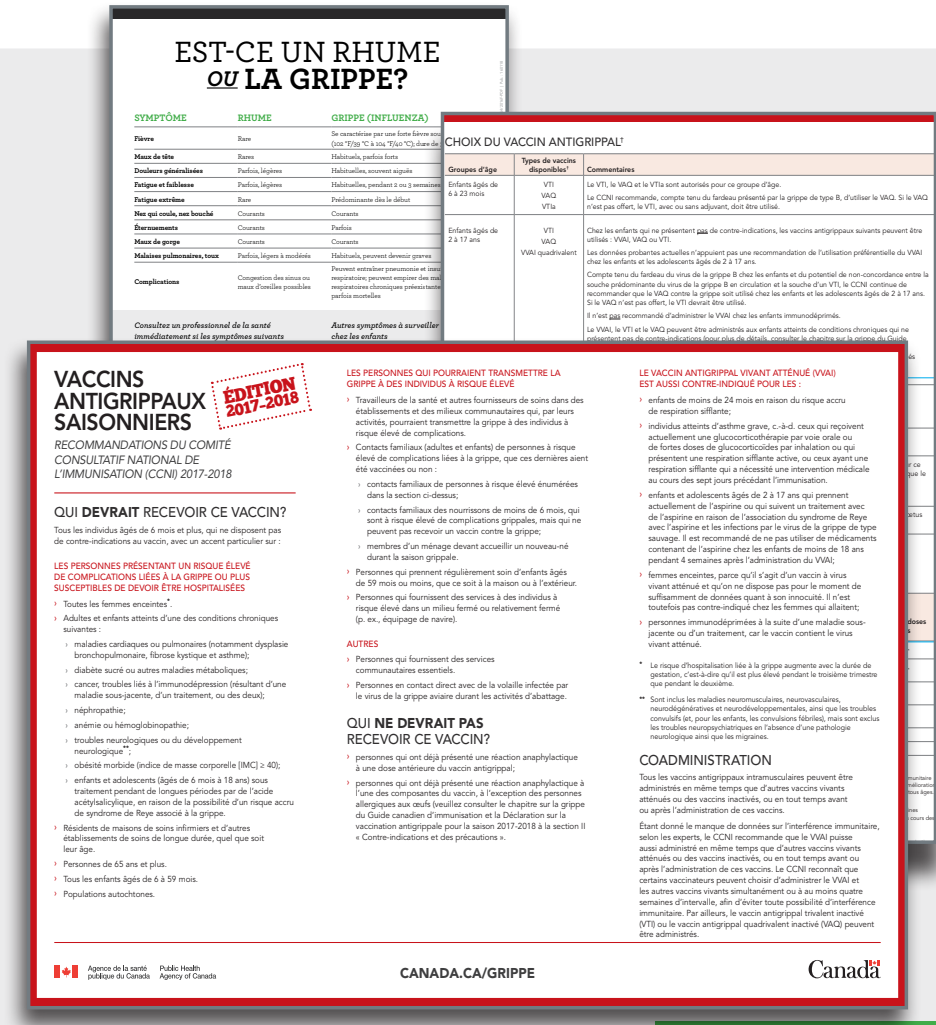

PASSEZ VOTRE COMMANDE AUJOURD'HUI. VISITEZ CANADA.CA/GRIPPE 